\title{
Statistical Properties of Carbon Nanostructures
}

\author{
Forrest H. Kaatz, Mesalands Community College, Tucumcari, NM, USA \\ Adhemar Bultheel, Dept. Computer Sci., KU Leuven, Heverlee, Belgium
}

\begin{abstract}
We look at modeling carbon nanostructures from a theoretical graph network view, where a graph has atoms at a vertex and links represent bonds. In this way, we can calculate standard statistical mechanics functions (entropy, enthalpy, and free energy) and matrix indices (Wiener Index) of finite structures, such as fullerenes and carbon nanotubes. The Euclidean Wiener Index (topographical index) is compared with its topological (standard) counterpart. For many of these parameters, the data have power law behavior, especially when plotted versus the number of bonds or the number of atoms. The number of bonds in a carbon nanotube is linear with the length of the nanotube, thus enabling us to calculate the heat of formation of capped $(5,5)$ and $(10,10)$ nanotubes. These properties are determined from atomic coordinates using MATLAB routines.
\end{abstract}

Keywords: fullerenes; carbon nanotubes; statistical mechanics; Wiener index; MATLAB

\section{Introduction}

Carbon has many allotropes (diamond, graphite, fullerenes, and carbon nanotubes, (CNTs)) that illustrate the amazing chemical and structural diversity of element number six. We consider the nanosized forms in our calculations; fullerenes and CNTs. Fullerenes were discovered in 1985 [1], carbon nanotubes in 1991 [2], and graphene in 2004 [3]. As a result of these groundbreaking discoveries, there are now literally thousands of scientists studying novel forms of carbon and their properties.

The first fullerene to be discovered was $C_{60}$, with a structure described as similar to that of a football (soccer ball) [1] with pentagonal cycles separated by hexagonal ones, see Figure 1A. This so-called isolated pentagon rule (IPR) is thought to stabilize the fullerene structure, since pentagonal bonds cost more energy than hexagonal ones. The smallest fullerene is $C_{20}$, consisting of 12 pentagons. In general, a fullerene has $n 3$-valent vertices with 12 pentagons and $(n / 2-10)$ hexagons, with $3 / 2 n$ carbon-carbon bonds. Experimentally, $C_{84}$ has been the largest fullerene [4] observed, but large 'carbon onions' [5] have been modeled theoretically. There are many isomers of the fullerene structures, so that several possible structures exist; however, most of these are not favored energetically.

Nanotubes were discovered in 1991 [2], as straight 'helical' coils of carbon, although filamentous carbon [6] was known prior to 1991. Thus a nanotube can be thought of as a rolled up sheet of graphene, with hemispheres of fullerenes at the ends. A commonly accepted growth mechanism [7,8] is that nanotubes form by catalytic action of transition metals, such as iron or cobalt, with a cap at one end and the open growth end eventually becoming closed after some aspect ratio is created. The nanotube analogue to $C_{60}$, the most common fullerene, seems to be a $(10,10)$ nanotube capped with hemispheres of $C_{240}$ [8], see Figure $1 \mathrm{~B}$ and $1 \mathrm{C}$.

Nanotubes are defined [9] by their 'chiral vector', or angle cut through the 2D layer of graphene, with

$$
n \mathbf{a}_{1}+m \mathbf{a}_{2} \equiv(n, m)
$$

where the indices $(n, m)$ describe the number of unit vectors in the hexagonal graphene honeycomb lattice. There are two common geometries, a 'zigzag' nanotube, with a chiral angle of $\theta=0^{\circ}$, and an 'armchair' nanotube with $\theta=30^{\circ}$, leaving a general chiral nanotube with $0^{\circ} \leq \theta \leq 30^{\circ}$. The chiral angle is given by:

$$
\theta=\tan ^{-1}[\sqrt{3} m /(m+2 n)]
$$

and the nanotube diameter is

$$
d_{t}=C_{h} / \pi=\sqrt{3} a_{c-c}\left(m^{2}+m n+n^{2}\right)^{1 / 2} / \pi
$$

where $a_{c-c}$ is the carbon-carbon nearest neighbor distance $(1.421 \AA)$ in graphite. Thus the $(10,10)$ nanotube is of the armchair variety. 


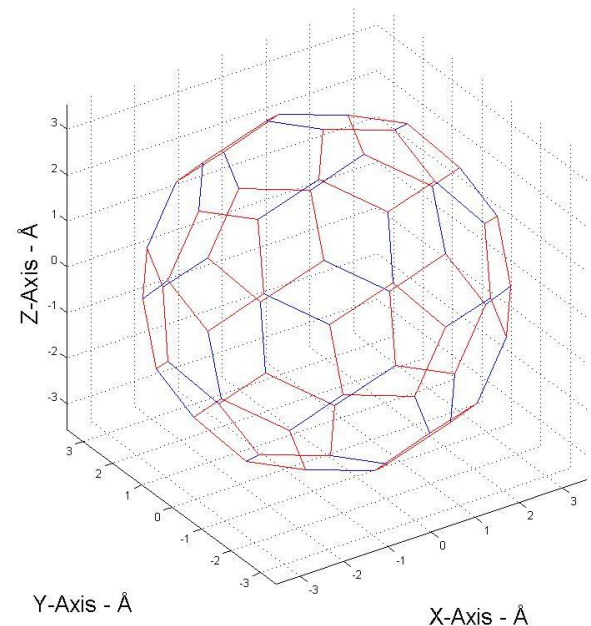

A. MATLAB plot of $C_{60}\left(I_{h}\right)$. Contrast indicates pentagonal cycles in the structure.

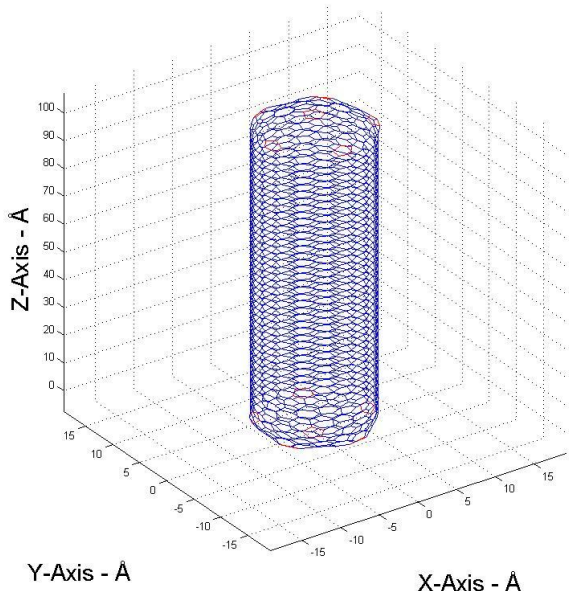

C. MATLAB plot of carbon nanotube $(10,10) 100 \AA$

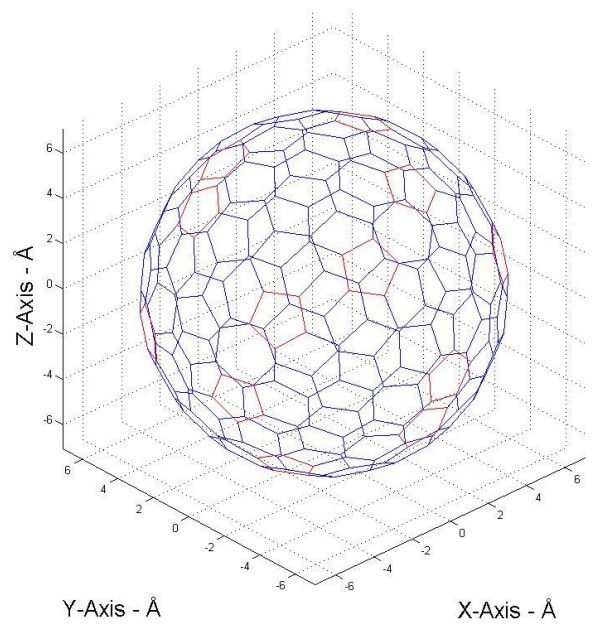

B. MATLAB plot of $C_{240}(5 v)$. Contrast indicates pentagonal cycles in the structure.

Figure 1: MATLAB plots 


\section{Methods}

We employ a graph-theoretical approach, where nodes represent atoms, and an edge represents a bond between sites, G $=(\mathrm{V}, \mathrm{E})$. We create a graph of the nanosized form of carbon by creating bonds (links) between nearest neighbors up to 1.3 times the shortest neighbor distance. These vary from 20 to 8360 atoms for fullerenes and nanotubes. An adjacency matrix is created and may exist in two forms. The standard form [10] is

$$
A= \begin{cases}H\left(r_{c}-r_{i j}\right) & i \neq j \\ 0 & i=j\end{cases}
$$

where the Heaviside step function $H\left(r_{c}-r_{i j}\right)=1$ if $r_{c}<1.3 * *$ shortest distance to $\left.r_{i j}\right)$, and $i$ and $j$ represent atomic sites, and $r_{c}$ is the cutoff value. Alternatively, we may consider the actual Euclidean distances in the adjacency matrix $[11,12]$, so that $H\left(r_{c}-r_{i j}\right)=e_{i j}$, the Euclidean distance between atoms.

The approach to modeling the free energy, enthalpy, and entropy has been discussed in the literature [13,14]. These can all be determined from the appropriate adjacency matrix. We also calculate one of the oldest indices, the Wiener index [15], as

$$
W=\frac{1}{2} \sum_{i=1}^{N} \sum_{j=1}^{N} d_{i j}
$$

where $N$ is the number of atoms and $d_{i j}$ is the shortest path distance between atoms $i$ and $j$. In the standard form, the distances between atoms $=1$, and in the Euclidean form [11], it is $e_{i j}$, so that we calculate $W_{E}$, the Euclidean 3D Wiener index. The collection of data starts from the atomic coordinates, and proceeds to calculating the adjacency matrix, and from it, all the results come from one MATLAB routine.

\section{Results}

In Figure 1, we show plots of finite nanocarbons, $C_{60}\left(I_{h}\right), C_{240}(5 v)$, and a $(10,10)$ nanotube. In our notation, the fullerene isomers are listed in parentheses as $I_{h}$ or $5 v$. The fullerenes and nanotubes have atoms with 3-fold coordination and the hexagonal and pentagonal cycles are shown in blue and red respectively. Once we have created the adjacency matrix, the statistical mechanics data can be calculated $[13,14]$. The partition function is:

$$
Z(G, \beta)=\operatorname{Tr}\left(e^{\beta A}\right)
$$

where $A$ is the adjacency matrix for the graph $G$, and $\beta=1 /\left(k_{B} T\right)$. At $T=300 K$, we have $\beta=38.68173 / \mathrm{eV}$. The entropy can be determined as

$$
S(G, \beta)=-k_{B} \sum_{j} \lambda_{j} p_{j}+k_{B} \ln (Z) \sum_{j} p_{j}
$$

where $\lambda_{j}$ is an eigenvalue of $A$ and

$$
p_{j}=\frac{e^{\beta \lambda_{j}}}{Z(G, B)}
$$

is the probability that the ensemble occupies a microstate $j$. The free energy is the natural logarithm of the partition function,

$$
F(G, \beta)=-\frac{\ln Z(G, \beta)}{\beta}
$$

and the enthalpy can be defined as follows:

$$
H(G, \beta)=-\frac{1}{Z(G, \beta)} \operatorname{Tr}\left(A e^{\beta A}\right) .
$$

We then plot the free energy, enthalpy, and entropy, per bond, versus the number of bonds in the nanocarbons. This results in plots with good power law [16] regression statistics as shown in Figure 2. We plot the data/bond versus the number of bonds for fullerenes and nanotubes. The distinction between (a) and (b) is that in (a), we have used an adjacency matrix with Euclidean distances, and in (b), we have used the standard adjacency matrix with zeros and ones. The bestfit equations in (a) have different leading coefficients, so that the entropy and enthalpy coincide (neglecting the sign difference) for small $(\approx 100$ atoms) nanocarbons, and since the slope is different, the plots diverge for larger structures. These quantities are divided by the number of bonds in the nanocarbon and plotted versus $N_{B}$, to give a power law plot. The asymptote of zero for large $N_{B}$ makes intuitive sense, since if we imagine the data/bond is finite, then as the number 


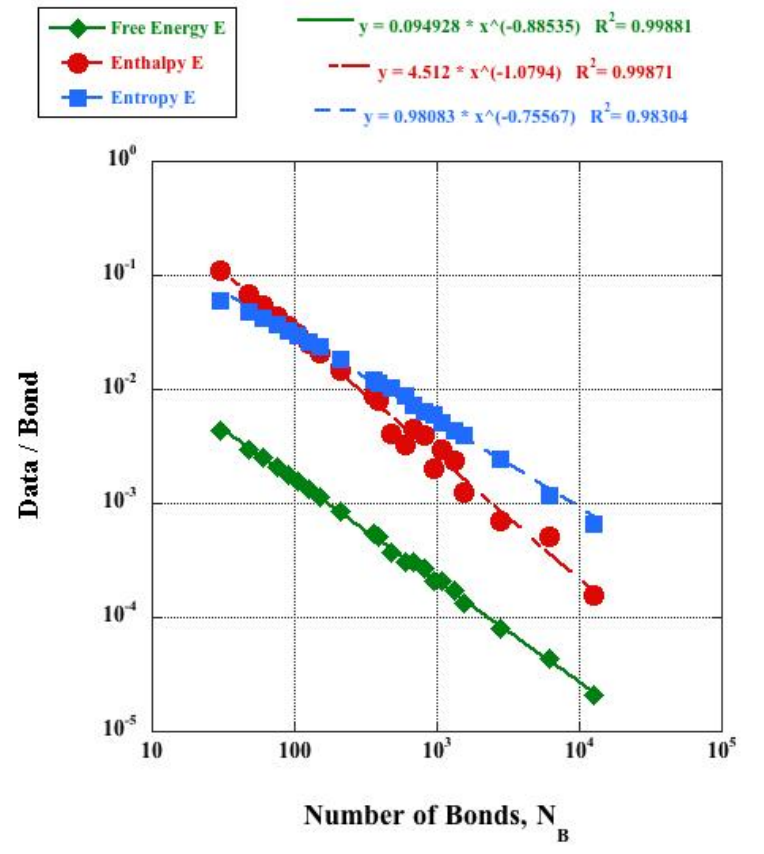

(a) Plots of the free energy, enthalpy, and entropy / bond versus the number of bonds. The data clearly exhibits power law character. This plot uses a Euclidean adjacency matrix.

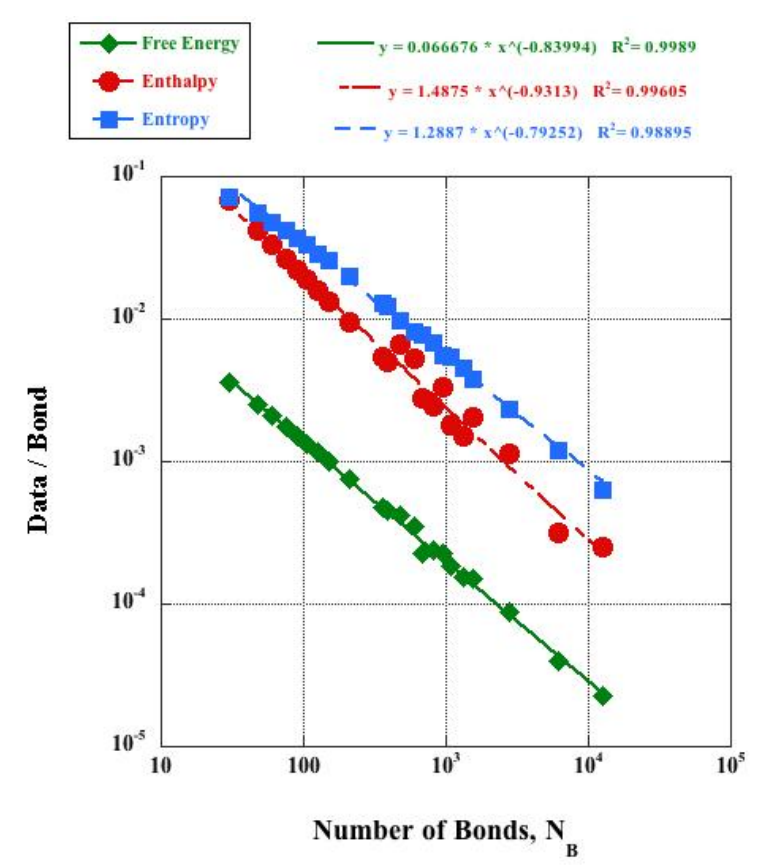

(b) Plots of the free energy, enthalpy, and entropy / bond versus the number of bonds. The data clearly exhibits power law character. This plot uses a standard adjacency matrix.

Figure 2: Power law plots for fullerenes and nanotubes.

\begin{tabular}{|c|l|}
\hline Structural Motif & $\varepsilon_{i} \cdot(\mathrm{kcal} / \mathrm{mol})$ \\
\hline$p p_{p}^{p}$ & 19.8 \\
\hline$p p_{p}^{h}$ & 17.6 \\
\hline$p p_{h}^{h}$ & 10.3 \\
\hline$p h_{p}^{p}$ & 15.7 \\
\hline$p h_{p}^{h}$ & 12.4 \\
\hline$p h_{h}^{h}$ & 7.8 \\
\hline$h h_{p}^{p}$ & 6.2 \\
\hline$h h_{p}^{h}$ & 4.7 \\
\hline$h h_{h}^{h}$ & 1.7 \\
\hline
\end{tabular}

Table 1: Heat of formation parameters for the nine structural motifs [17]. 


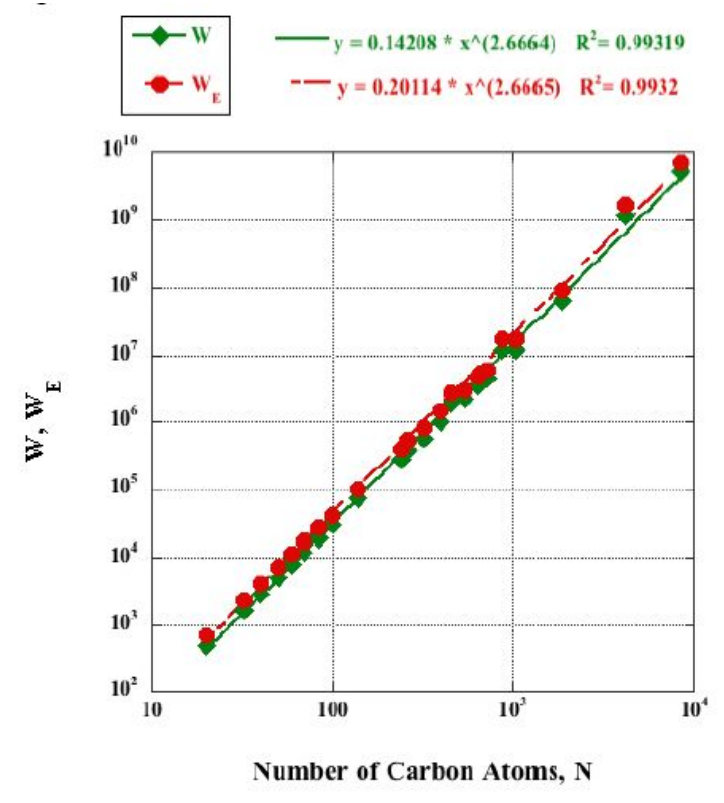

Figure 3: Power law plot of the Wiener Index (standard and Euclidean) for fullerenes and nanotubes versus the number of atoms, $N$.

of bonds becomes large, we have zero as a limit. Note that the free energy and enthalpy have their signs reversed to allow them to be plotted.

For the fullerenes and nanotubes, we can calculate the energy of formation based on the types of bonds [17] in the structures, see Table 1 . We use three types of bonds, as $h h, h p$, and $p p$, indicating hexagonal or pentagonal edges, and then further subdivided as to whether the opposite ends of the bond meets a hexagonal or pentagonal cycle. This gives a total of nine bonds and the heat of formation can be calculated as:

$$
\Delta H_{f}=\sum_{i}^{\text {motifs }} \varepsilon_{i} n_{i}
$$

where $\varepsilon_{i}$ is the energy contribution of each structural motif (nine) and $n_{i}$ is the number of bonds associated with that motif. Since fullerenes have been analyzed extensively [18-20], we show $\Delta H_{f}$ versus length in Angstroms for two of the more common nanotubes, the $(5,5)$ and $(10,10)$ armchair varieties. For nanotubes, the heat of formation includes the energy to create the cap and then the length of the tube. We plot these results in Figure 3. The $(5,5)$ nanotubes contain a cap of $C_{60}$ which has $60 p h_{h}^{h}$ bonds and $30 h h_{p}^{p}$ bonds to give $\Delta H_{f}=654 \mathrm{kcal} / \mathrm{mol}$. As the length of the nanotube increases, we add $h h_{h}^{h}$ bonds at $1.7 \mathrm{kcal} / \mathrm{mol}$ for each bond. The $(10,10)$ nanotubes have a cap of $C_{240}$ (see Figure 1B) [7], which has $60 p h_{h}^{h}$ bonds, $60 h h_{p}^{h}$ bonds, and $240 h h_{h}^{h}$ bonds, to give $\Delta H_{j}=1158 \mathrm{kcal} / \mathrm{mol}$. Again, as the length of the nanotube increases, we add $h h_{h}^{h}$ bonds at $1.7 \mathrm{kcal} / \mathrm{mol}$ for each bond. From the graph, we see that since the $(10,10)$ tube is larger, the slope is $41.63 \mathrm{kcal} / \mathrm{mol}$ per $\AA$ length versus $20.689 \mathrm{kcal} / \mathrm{mol}$ per $\AA$ length for the $(5,5)$ nanotube. For the $(10,10)$ nanotubes, this gives a cap energy of $64 \mathrm{eV}$ and a length energy of $1.8 \mathrm{eV} / \AA$. These data are in good agreement [7] ( $40 \mathrm{eV}$ and $0.74 \mathrm{eV} / \AA ̊$ ) with estimated measurements.

Since we are using a graph-theoretical language, we also calculate the Wiener index for fullerenes and nanotubes, based on the coordinate information in MATLAB. We note that for fullerenes, the Wiener index of $C_{60}(\mathrm{Ih})=8340$ has been known since 1992 [21], and since then some additional results have been calculated [22-24]. The Wiener index for armchair and zigzag nanotubes has been known since 2004 [25,26]. In Table 2, we list some of the Wiener indices for the fullerenes we investigate, and also the $(5,5)$ and $(10,10)$ nanotubes [54]. The Wiener Index for the fullerenes up to $C_{84}$ agree with previous [22] results, and we add new results up to C720 and also the Euclidean indices for all nanocarbons. In Figure 4, we plot the Wiener index versus $N$, the number of carbon atoms, to give a power law relationship. We note that for the fullerenes, the Wiener index depends on the structure, so each isomer has a different value for the index. Also, for the nanotubes, the original calculation $[25,26]$ of the Wiener Index did not include caps on the ends, so a direct comparison is not possible. Our modeling is for nanotubes up to $500 \AA$ in length, with caps at both ends. 


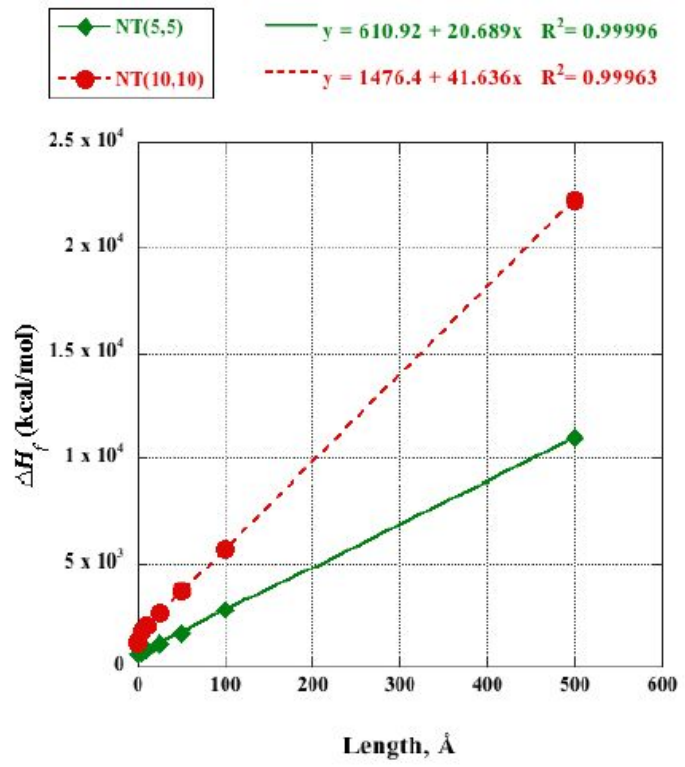

Figure 4: Heat of formation for $(5,5)$ and $(10,10)$ armchair nanotubes versus length in Angstroms.

\begin{tabular}{|c|c|c|c|c|c|}
\hline$N$ & $N_{B}$ & Nanostruct & Wiener Index $(W)$ & Euclid. Wiener Ind. $\left(W_{E}\right)$ & Ave Bond $(\AA)$ \\
\hline 20 & 30 & $C_{20}\left(I_{h}\right)$ & 500 & 710.02423 & 1.4200 \\
\hline 32 & 48 & $C_{32}\left(D_{3 d}\right)$ & 1696 & 2408.3699 & 1.4200 \\
\hline 40 & 60 & $C_{40}\left(T_{d}\right)$ & 3000 & 4260.0679 & 1.4200 \\
\hline 50 & 75 & $C_{50}\left(D_{3}\right)$ & 5275 & 7490.6239 & 1.4200 \\
\hline 60 & 90 & $C_{60}\left(I_{h}\right)$ & 8340 & 11917.75156 & 1.4320 \\
\hline 70 & 105 & $C_{70}\left(D_{5 h}\right)$ & 12375 & 17672.68417 & 1.4307 \\
\hline 84 & 126 & $C_{84}\left(D_{2}\right)$ & 19646 & 28041.35179 & 1.4303 \\
\hline 240 & 360 & $C_{240}$ & 277440 & 392329.2316 & 1.4187 \\
\hline 540 & 810 & $C_{540}$ & 2119320 & 2994979.407 & 1.4190 \\
\hline 720 & 1080 & $C_{720}$ & 4352340 & 6162553.116 & 1.4207 \\
\hline 100 & 150 & $(5,5) 5$ & 30580 & 42310.62308 & 1.3879 \\
\hline 140 & 210 & $(5,5) 10$ & 72860 & 101743.2403 & 1.3962 \\
\hline 260 & 390 & $(5,5) 25$ & 383700 & 541217.5044 & 1.4059 \\
\hline 460 & 690 & $(5,5) 50$ & 1899100 & 2689448.448 & 1.4107 \\
\hline 880 & 1320 & $(5,5) 100$ & 12350940 & 17526597.26 & 1.4150 \\
\hline 4120 & 6180 & $(5,5) 500$ & 1186916820 & 1685572294.8 & 1.4164 \\
\hline 320 & 480 & $(10,10) 5$ & 569680 & 804021.9498 & 1.4156 \\
\hline 400 & 600 & $(10,10) 10$ & 997120 & 1410161.755 & 1.4165 \\
\hline 640 & 960 & $(10,10) 25$ & 3341840 & 4737709.644 & 1.4178 \\
\hline 1040 & 1560 & $(10,10) 50$ & 12327040 & 17496098.43 & 1.4193 \\
\hline 1860 & 2790 & $(10,10) 100$ & 62759375 & 89129458.54 & 1.4187 \\
\hline 8360 & 12540 & $(10,10) 500$ & 5046069000 & 7169083944.2 & 1.4199 \\
\hline
\end{tabular}

Table 2: $N$ is the number of atoms in the nanostructure, fullerenes are denoted by $C_{N}$ and nanotubes by their chiral indices followed by their length in Angstroms. The topological Wiener Index is $W$, and the Euclidean Wiener Index is $W_{E}$. The bond length is in Angstroms. 


\section{Conclusion}

In summary, we have determined the power law behavior of the free energy, enthalpy, entropy, and atomic displacement of nanocarbons consisting of 20-8360 atoms. We use atomic coordinates to calculate the Wiener index for fullerenes and nanotubes. There are some minor distinctions when using the Euclidean Wiener index, which we have included for completeness. The heat of formation of nanotubes follows a linear relationship with length in agreement with known data.

We have outlined procedures applicable to modeling finite carbon nanostructures using only the 3D coordinates of the structures. These methods will allow others to investigate similar types of models and we encourage the understanding of nano-geometries as we move into the 21 st century.

\section{References}

[1] Kroto, H.W.; Heath, J.R.; O’Brien, S.C.; Curl, R.F. Smalley, R.E. Nature 1985, 318, 162- 163.

[2] Iijima, S. Nature 1991, 354, 56-58.

[3] Novoselov, K.S.; Geim, A.K.; Morozov, S.V.; Jiang, D.; Zhang,Y.; Dubonos, S.V.; Grigorieva,I.V.; Firsov, A.A. Science 2004 306, 666-669.

[4] Halasinshi, T.M.; Ruiterkamp, R.; Salama, F.; Foing, B.H.; Ehrenfreund, R. Fullerenes, Nanotubes and Carbon Nanostructures 2011, 19(5), 398-409.

[5] Lin, Y.L.; Nori, F. Phys. Rev B 1994, 49(7), 5020-5023.

[6] Baker, R.T.K. Carbon 1989, 27, 315-327.

[7] Thess, A. et al. Science 1996, 273, 483-487.

[8] Jin, C.; Suenaga, K.; Iijima, S. ACS Nano, 2008, 2(6), 1275-1279.

[9] Dresselhaus, M.S.; Eklund, P.C. Adv. Phys., 2000, 49(6), 705-814.

[10] Estrada, E.; Hatano., N. Chem. Phys. Lett., 2010, 486, 166-170.

[11] Nikolic, S.; Trinajstic, N.; Mihalic, Z.; Carter, S. Chem. Phys. Lett., 1991, 179, 21-28.

[12] Vodopivec, A.; Kaatz, F.H.; Mohar, B. J. Math. Chem., 2010, 47, 1145-1153.

[13] Estrada,E.; Hatano, N. Chem. Phys. Lett., 2007, 439, 247-251.

[14] Kaatz, F.H.; Estrada, E.; Bultheel, A.; Sharrock, N. Physica A, 2012, 391, 2957-2963.

[15] Wiener, H. J. Am. Chem. Soc. 1947 69, 17-20.

[16] Clauset, A.; Shalizi, C.R.; Newman, M.E.J. SIAM Rev., 2009, 51, 661-703.

[17] Alcami, M.; Sanchez, G.; Diaz-Tendero, S.; Wang, Y.; Martin, F. J. Nanosci. Nantechnol., 2007, 7, 1329-1338.

[18] Rojas, A.; Marinez, M.; Amador, P.; Torres, L.A. J. Phys. Chem. B, 2007, 111, 9031-9035.

[19] Cioslowski, J.; Rao, N.; Moncrieff, D. J. Am. Chem. Soc., 2000, 122, 8265-8270.

[20] Lair, S.L.; Herndon, W.C.; Murr, L.E.; Quinones, S.A. Carbon, 2006, 44, 447-455.

[21] Ori, O.; D’Mello, M. Chem. Phys. Lett., 1992, 197, 49-54.

[22] Balasubramanian, K. J. Phys. Chem., 1995, 99, 10785-10796.

[23] Babic, D.; Klein, D.J.; Lukovits, I.; Nikolic, S.; Trinajstic, N. Internat. J. Quantum Chem., 2002, 90, 166-176.

[24] Fowler, P.W. Croatica Chem. Acta, 2002, 75(2), 401-408.

[25] John, P.E.; Diudea, M.V. Croatica Chem. Acta,2004, 77(1-2), 127-132.

[26] Diudea, M.V.; Stefu, M.; Parv, B.; John, P.E. Croatica Chem. Acta, 2004, 77(1-2), 111-115. 